\title{
PRODUÇÃO DE CONCENTRADO DE MAGNETITA POR JIGAGEM A PARTIR DO REJEITO DE ROCHA FOSFÁTICA
}

\author{
R. S. TOMÁZ ${ }^{1}$, A. C. SILVA ${ }^{2 *}$, E. M. S. SILVA e D. N. SOUSA ${ }^{1}$ \\ ${ }^{1}$ Instituto Federal Goiano \\ ${ }^{2}$ Universidade Federal de Goiás \\ ancarsil@ufg.br
}

Artigo submetido em novembro/2015 e aceito em dezembro/2015

DOI: 10.15628/holos.2015.3715

\section{RESUMO}

A magnetita $\left(\mathrm{Fe}_{3} \mathrm{O}_{4}\right)$ é um óxido magnético de ferro natural formado por íons $\mathrm{Fe}^{+2}$ e $\mathrm{Fe}^{+3}$. É o mais comum mineral fortemente magnético, estando presente em pequenas quantidades em quase todas as rochas e também nos meteoritos. A nanotecnologia está largamente presente em todo nosso dia a dia. É possível encontrar diferentes aplicações nas mais diversas áreas da indústria: Eletroeletrônicos, metalurgia, cerâmicos, tintas, tecidos, indústria de polímeros, medicina entre outras. Atualmente a Anglo American Fosfatos do Brasil não produz magnetita, mas possui uma produção de rejeito de $2 \mathrm{Mt}$ (milhões toneladas) com teores de magnetita por volta de 40\%, na granulometria de $2 \mathrm{~mm}$, sendo esse material enviado integralmente para a barragem de rejeito. $\mathrm{O}$ aproveitamento desse material, dada uma recuperação de $60 \%$, traria um crescimento de
78,5 milhões de dólares no PIB do estado. A maior parte da produção de ferro do Brasil é feita por flotação, sendo que tal tipo de produção prejudica a utilização da magnetita, especificamente, para a produção de ferrofluidos nanomagnéticos, devido a enorme quantidade de produtos químicos, principalmente surfactantes, envolvidos na etapa de flotação. Contudo o objetivo desse trabalho é obter um concentrado de magnetita proveniente do rejeito da produção de rocha fosfática por jigagem, para que a mesma possa ser usada na produção de ferrofluidos. Os resultados da utilização do jigue como separador dos minerais são satisfatórios na produção de magnetita para ferrofluidos como coproduto da produção de concentrado de rocha fosfática.

PALAVRAS-CHAVE: areia; jigagem, magnetita; rocha fosfática.

\section{PRODUCTION OF MAGNETITE CONCENTRATE BY JIGGING FROM PHOSPHATE ROCK}

\begin{abstract}
Magnetite $\left(\mathrm{Fe}_{3} \mathrm{O}_{4}\right)$ is a magnetic iron oxide formed by natural ions $\mathrm{Fe}^{+2}$ and $\mathrm{Fe}^{+3}$. It is the most common mineral strongly magnetic, being present in small amounts in almost all rocks and also in meteorites. Nanotechnology is widely present throughout our daily lives. You can find different applications in several areas of the industry: consumer electronics, metallurgy, ceramics, paints, fabrics, polymer industry, medicine and others. Currently Anglo American phosphates in Brazil does not produce magnetite, but has a production tailings $2 \mathrm{Mt}$ (million tonnes) with magnetite content around $40 \%$, the grain size of $2 \mathrm{~mm}$, this material fully sent to the tailings dam . The use of this material, with a
\end{abstract}

recovery of $60 \%$ would bring an increase of 78.5 million in the state's GDP. Most of the iron production in Brazil is made for flotation, this kind of production preclude the use of magnetite, specifically for the production of nanomagnetics fluids, due to the large amount of chemicals, mainly surfactants, involved in stage of flotation. However our aim is to get a magnetite concentrate from the tailings of phosphate rock production by jigging, so that it can be used in the production of nanomagnetics fluids. The results of the use of jig as a separator minerals are suitable for the production of nanomagnetics fluids as coproduct of phosphate rock concentrate production.

KEYWORDS: : jigging; magnetite; phosphate rock; sand. 


\section{INTRODUÇÃO}

A magnetita (Fe3O4) é um óxido magnético de ferro natural formado por íons Fe+2 e Fe+3. É o mais comum mineral fortemente magnético, estando presente em pequenas quantidades em quase todas as rochas e também nos meteoritos. A presença de magnetita em lavas vulcânicas que formam o assoalho oceânico permite que se estude o campo magnético terrestre da época de seu resfriamento e suas propriedades são conhecidas desde os tempos da Grécia Antiga. Em países onde não ocorre a hematita, a magnetita é utilizada como importante fonte de ferro. (BRASIL, 2014).

Devido a sua magnetização espontânea e estabilidade química, nanopartículas de magnetita (geralmente de $10 \mathrm{~nm}$ ) são muito utilizadas na preparação de ferrofluidos (mistura coloidal de um solvente com nanopartículas magnéticas). O tamanho característico para as partículas esféricas de magnetita se tornarem monodomínios magnéticos é de $12,4 \mathrm{~nm}$, portanto esses ferrofluidos geralmente são formados por partículas de magnetita no estado de monodomínio magnético. Essas partículas são recobertas por surfactantes para impedir a aglomeração.

A nanotecnologia está largamente presente em todo nosso dia a dia. O tema vem ganhando respaldo a medida que técnicas de caracterização e identificação de novos nanomateriais vem sendo lançadas pelo mercado. Graças ao potencial e versatilidade da nanotecnologia ela é considerada uma tecnologia transversal. É possível encontrar diferentes aplicações nas mais diversas áreas da indústria: eletroeletrônicos, metalurgia, cerâmicos, tintas, tecidos, indústria de polímeros, medicina entre outras.

Atualmente a Anglo American Fosfatos do Brasil não produz magnetita, mas possui uma produção de rejeito de $2 \mathrm{Mt}$ (milhões toneladas) com teores de barita de $6 \%$ e magnetita por volta de $40 \%$, na granulometria de $2 \mathrm{~mm}$, sendo esse material enviado integralmente para a barragem de rejeito. O aproveitamento desse material, dada uma recuperação de $60 \%$, traria um crescimento de 78,5 milhões de dólares no PIB (Produto Interno Bruto) do estado.

Atualmente, a maioria das rotas de síntese usada na obtenção de nanopartículas de magnetita apresenta algum tipo de inconveniente, geralmente associado ao meio de síntese em solução aquosa, como o alto índice de aglomerados e a remoção de surfactantes.

A maior parte da produção de ferro do Brasil, seja beneficiando hematita quanto magnetita, é feita por flotação, sendo que tal tipo de produção prejudica a utilização da magnetita, especificamente, para a produção de ferrofluidos, devido a enorme quantidade de produtos químicos, principalmente surfactantes, envolvidos na etapa de flotação. (Costa, 2013)

Os métodos de separação gravítica são utilizados para tratar uma grande variedade de minerais, com grande variedade de densidades, que vão desde os sulfetos de metais pesados tais como a galena $(7,5 \mathrm{~g} / \mathrm{cm} 3)$ a carvão $(0,85 \mathrm{~g} / \mathrm{cm} 3)$, e grande variedade de tamanhos de partículas (Lins, 2010).

A concentração densitária perdurou como método de processamento mineral dominante por cerca de 2000 anos e foi somente no século 20 que sua importância declinou, com o 
desenvolvimento de processos como a flotação e a concentração magnética que permitira o tratamento seletivo de minérios complexos e de baixos teores (Wills, 2006).

A jigagem é um processo de separação hidráulica que consiste da repetida expansão e contração vertical de um leito de partículas pelo movimento pulsante de água. $O$ resultado desse movimento é a estratificação do leito, onde se tem a separação dos materiais por densidade em ordem crescente do topo à base (Sampaio e Tavares, 2005).

Como a utilização da flotação para a concentração de magnetita não é interessante devido à restrição da sua utilização, o enriquecimento do rejeito pela jigagem para a extração desse mineral se justifica devido à diferença de massa específica dos minerais envolvidos $(5,1 \mathrm{~g} / \mathrm{cm} 3$ para a magnetita e 3,1 g/cm3 para a apatita), aliado a simplicidade de operação do equipamento e pelo seu baixo custo de operação.

A retirada da magnetita do processo de produção de fosfato trás três grandes ganhos: o primeiro seria a redução de material enviado para os passos de flotação da barita e apatita, o segundo é a geração de um material que poderá ser utilizado na produção de ferrofluidos e o terceiro seria a redução de material enviado para a barragem de rejeito, evitando assim a construção de novas barragens e reduzindo os impactos ambientais.

Com a dificuldade em se obter materiais provenientes do rejeito das mineradoras da região de Catalão, para obtenção dos dados experimentais foi sugerido a substituição das substâncias mistas por areia (quartzo), já que a mesma possui uma boa diferença de densidade e densidade próxima aos minerais provenientes do minério de rocha fosfática, é de fácil obtenção, e possui baixo custo.

Contudo o objetivo desse trabalho é obter um concentrado de magnetita proveniente do rejeito da produção de rocha fosfática por jigagem, para que a mesma possa ser usada na produção de ferrofluidos.

Os resultados da utilização do jigue como separador dos minerais são satisfatórios, sendo o próximo passo a utilização de material vindo diretamente do rejeito das mineradoras de Catalão para exploração dos resultados e analisarmos a viabilidade da produção de magnetita para ferrofluidos como coproduto da produção de concentrado de rocha fosfática.

\section{METODOLOGIA}

O trabalho experimental foi dividido em três etapas. Na primeira, as amostras de quartzo e magnetita foram bitoladas granulometricamente por peneiramento a úmido. Na segunda etapa foi efetuada uma separação magnética em ambos minerais para a retirada de contaminantes no quartzo e de partículas mistas na magnetita. Na terceira etapa foram realizados ensaios de jigagem.

\subsection{Peneiramento do material}

As amostras minerais a serem utilizadas nos ensaios de jigagem foram quarteadas para obtenção de amostras de $400 \mathrm{~g}$ e peneiradas via peneiramento úmido de modo a obter amostras bitoladas de 0,180 $\mu \mathrm{m}, 150 \mu \mathrm{m}$ e $106 \mu \mathrm{m}$. O tempo de peneiramento adotado foi de 15 minutos. 


\subsection{Separação magnética da amostra}

Para a obtenção de amostras de mais alta pureza, todo material peneirado foi submetido a uma separação magnética utilizando-se um ímã de terras raras de 2,0 G. Para a composição das amostras foram misturados o quartzo e a magnetita na proporção de $50 \%$ de cada em mesma granulometria. Após homogeneização alíquotas de $100 \mathrm{~g}$ foram enviadas para os testes de jigagem.

\subsection{Ensaios de jigagem}

Os ensaios foram realizados empregando-se o Jigue Denver de labora, modelo SJ-1015, de fabricação pela Engendrar (Figura 1). Este equipamento possui o crivo fixo, o impulso da água é proporcionado pelo movimento do diafragma. Para a realização dos testes o jigue operou com uma vazão fixa de água e foram escolhidas duas variáveis operacionais: a granulometria da alimentação e a frequência dos pulsos.

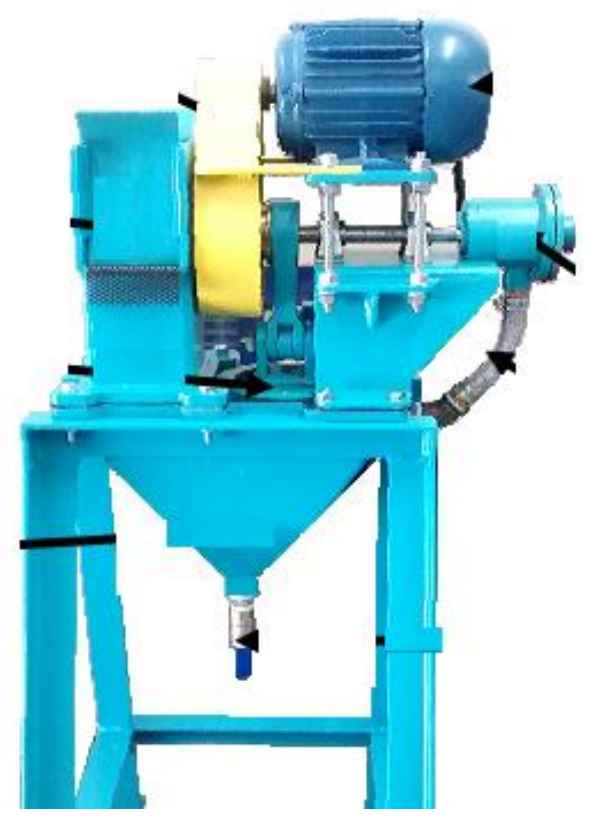

(a)

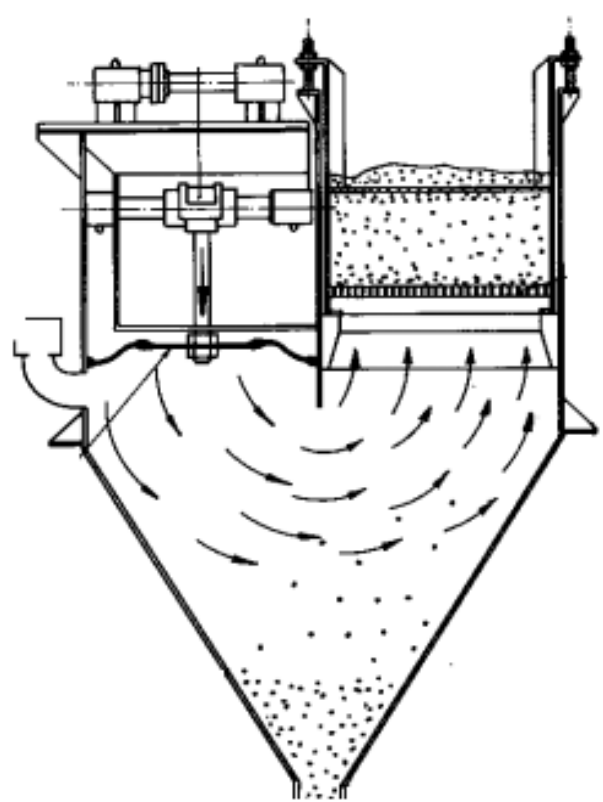

(b)

Figura 1: a) foto do Jigue Denver, modelo SJ-1015, b) desenho esquemático do jigue de diafragma. Fonte: ENGENDRAR

Foram feitas três conjuntos de testes, dividindo as amostras em três frações granulométricas: 0,180 $\mu \mathrm{m}, 150 \mu \mathrm{m}$ e $106 \mu \mathrm{m}$. Para variação da frequência de pulsos utilizou-se um inversor de frequência acoplado ao motor do equipamento. Este inversor controlava a rotação do motor do jigue de 30 a 350 RPM (Rotações por Minuto). Para os ensaios de jigagem foram utilizadas as frequências de 100, 220 e 330 RPM.

Os ensaios foram realizados colocando-se as amostras no jigue com fluxo de água contínuo e na frequência de pulsos desejada. O material leve (transbordado) e o pesado (afundado) eram recolhidos, filtrados, secos em estufa e pesados. Após a pesagem, o material (leve e pesado) era 
submetido a separação magnética, obtendo-se assim um concentrado magnético e outro não magnéticos. Todos os testes foram realizados em triplicata.

\section{RESULTADOS E DISCUSSÃO}

A Tabela 1 apresenta os resultados da jigagem de quartzo e magnetita bitolado em $180 \mu \mathrm{m}$. Nota-se que com o aumento da frequência dos pulsos o teor de quartzo no afundado diminuiu. A Figura 2 apresenta a diminuição da massa de quartzo no afundado com o aumento dos pulsos do jigue.

Tabela 1 Resultados obtidos na granulometria de $180 \mu \mathrm{m}$ (80\#).

\begin{tabular}{c|c|c|c|c|c|c}
\hline \multirow{2}{*}{$\begin{array}{c}\text { Frequência } \\
\text { (RPM) }\end{array}$} & \multicolumn{2}{|c|}{ Amostra (g) } & \multicolumn{2}{c|}{ Material afundado (g) } & \multicolumn{2}{c}{ Enriquecimento (\%) } \\
\cline { 2 - 6 } & Quartzo & Magnetita & Quartzo & Magnetita & Quartzo & Magnetita \\
\hline 100 & 53,16 & 51,00 & 43,40 & 50,81 & $-2,89$ & 4,97 \\
\hline 220 & 50,89 & 51,26 & 38,24 & 51,90 & $-8,38$ & 7,40 \\
\hline 330 & 51,70 & 50,67 & 33,38 & 49,74 & $-13,53$ & 10,35 \\
\hline
\end{tabular}

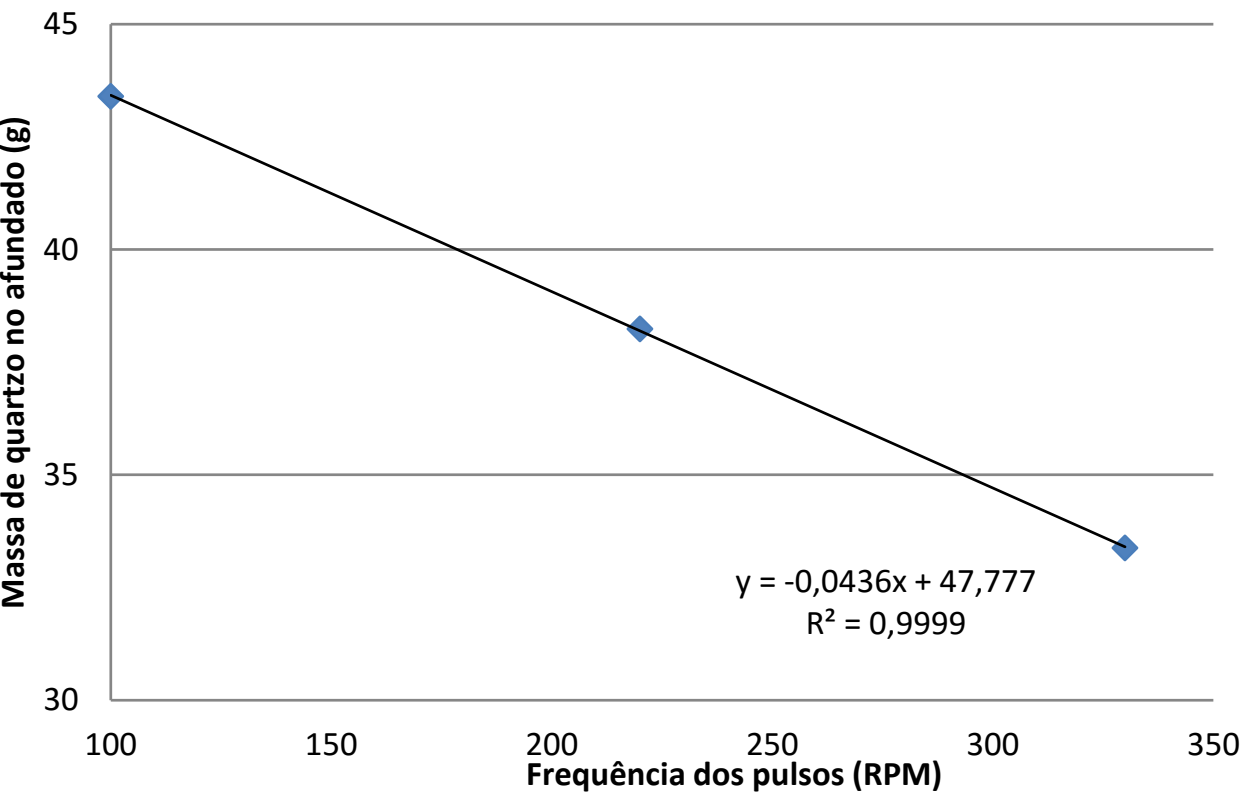

Figura 2: Massa de quartzo no material afundado em função da frequência dos pulsos do jigue para granulometria de $180 \mu \mathrm{m}(80 \#)$.

A Tabela 2 apresenta os resultados da jigagem de quartzo e magnetita bitolado em $150 \mu \mathrm{m}$ (100\#). Novamente o aumento da frequência dos pulsos gerou uma diminuição no teor de quartzo no afundado. Contudo, diferente do esperado pela literatura, os resultados encontrados para a granulometria de $100 \#$, mais fina que a anterior, foram melhores com relação às massas de quartzo e magnetita no afundado. A Figura 3 apresenta a diminuição da massa de quartzo no afundado com o aumento dos pulsos do jigue.

A Tabela 3 apresenta os resultados da jigagem de quartzo e magnetita bitolado em $106 \mu \mathrm{m}$ (150\#). O aumento da frequência dos pulsos gerou uma diminuição no teor de quartzo no afundado, sendo que a diminuição na granulometria dos dois minerais favoreceu ainda mais a 
separação dos mesmos. A Figura 4 apresenta a diminuição da massa de quartzo no afundado com o aumento dos pulsos do jigue.

Tabela 2: Resultados obtidos na granulometria de $150 \mu \mathrm{m}$ (100\#)

\begin{tabular}{c|c|c|c|c|c|c}
\hline \multirow{2}{*}{$\begin{array}{c}\text { Frequência } \\
\text { (RPM) }\end{array}$} & \multicolumn{2}{|c|}{ Alíquotas (g) } & \multicolumn{2}{c}{ Material afundado (g) } & \multicolumn{2}{c}{ Enriquecimento (\%) } \\
\cline { 2 - 7 } & Quartzo & Magnetita & Quartzo & Magnetita & Quartzo & Magnetita \\
\hline 100 & 52,49 & 50,74 & 33,80 & 50,30 & $-8,96$ & 10,65 \\
\hline 220 & 51,36 & 50,06 & 32,80 & 49,71 & $-10,15$ & 10,88 \\
\hline 330 & 55,03 & 51,75 & 31,90 & 51,29 & $-11,22$ & 13,19 \\
\hline
\end{tabular}

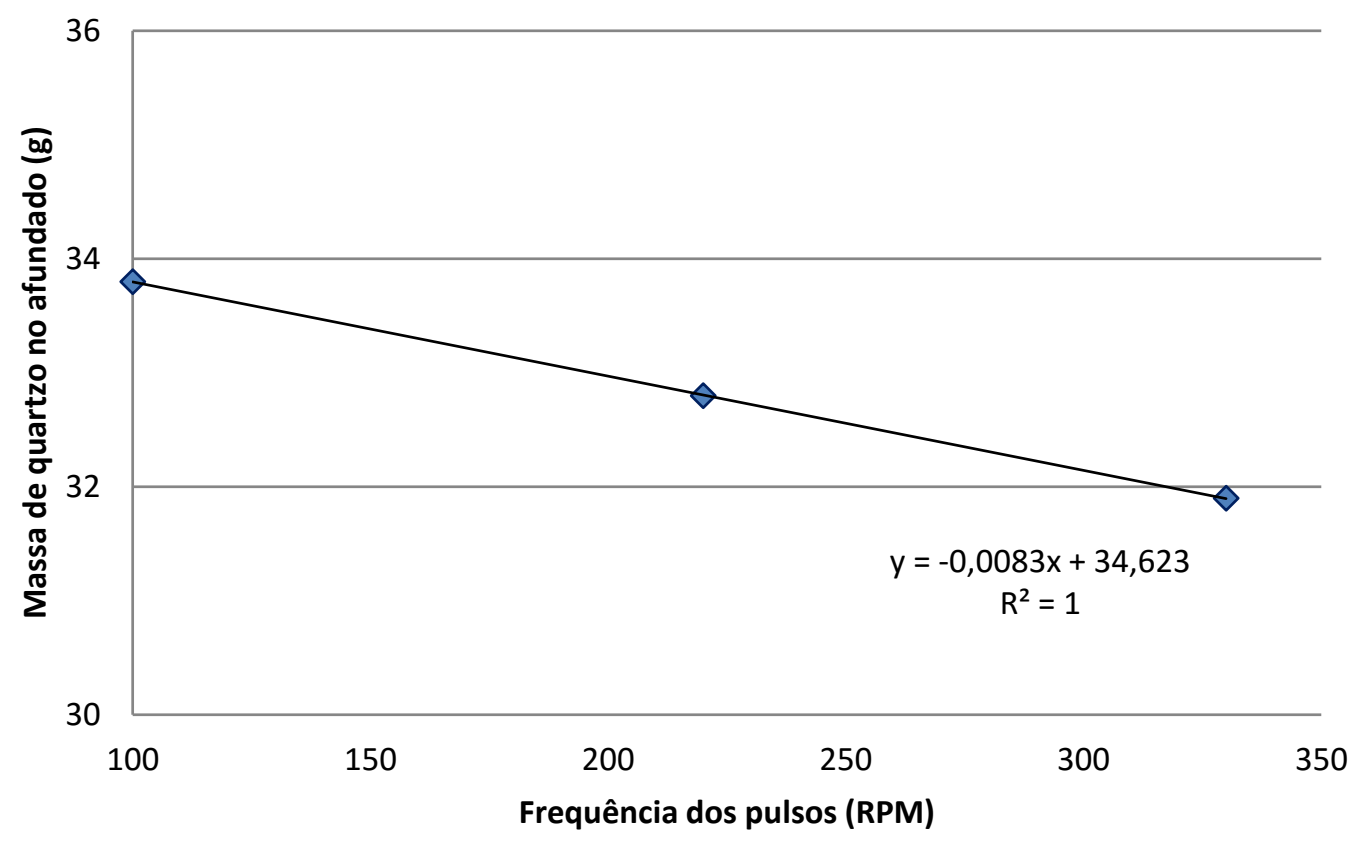

Figura 3: Massa de quartzo no material afundado em função da frequência dos pulsos do jigue para granulometria de $150 \mu \mathrm{m}(100 \#)$

Tabela 3: Resultados obtidos na granulometria de $106 \mu \mathrm{m}$ (150\#)

\begin{tabular}{c|c|c|c|c|c|c}
\hline \multirow{2}{*}{$\begin{array}{c}\text { Frequência } \\
\text { (RPM) }\end{array}$} & \multicolumn{2}{|c|}{ Alíquotas (g) } & \multicolumn{2}{c}{ Material afundado (g) } & \multicolumn{2}{c}{ Enriquecimento (\%) } \\
\cline { 2 - 7 } & Quartzo & Magnetita & Quartzo & Magnetita & Quartzo & Magnetita \\
\hline 100 & 53,23 & 50,79 & 29,20 & 50,29 & $-12,09$ & 14,43 \\
\hline 220 & 52,19 & 51,45 & 26,06 & 50,48 & $-16,05$ & 16,31 \\
\hline 330 & 51,61 & 52,77 & 21,46 & 50,90 & $-21,84$ & 19,79 \\
\hline
\end{tabular}

Os resultados dos ensaios mostram que há um enriquecimento mássico da magnetita e um decréscimo do quartzo no afundado. Com isso tem-se que o procedimento de jigagem possui capacidade de melhorar o material fazendo a separação destes minerais.

As amostras apresentaram uma clara tendência de aumento do enriquecimento para as frações mais finas. Deve-se ressaltar que mesmo nas frações mais grossas houve uma retirada positiva de materiais leves, tendo visto um enriquecimento do elemento de interesse (magnetita) na parte afundada, com a diminuição das partículas de quartzo no material afundado, como visto nas figuras 3 a 5 . 


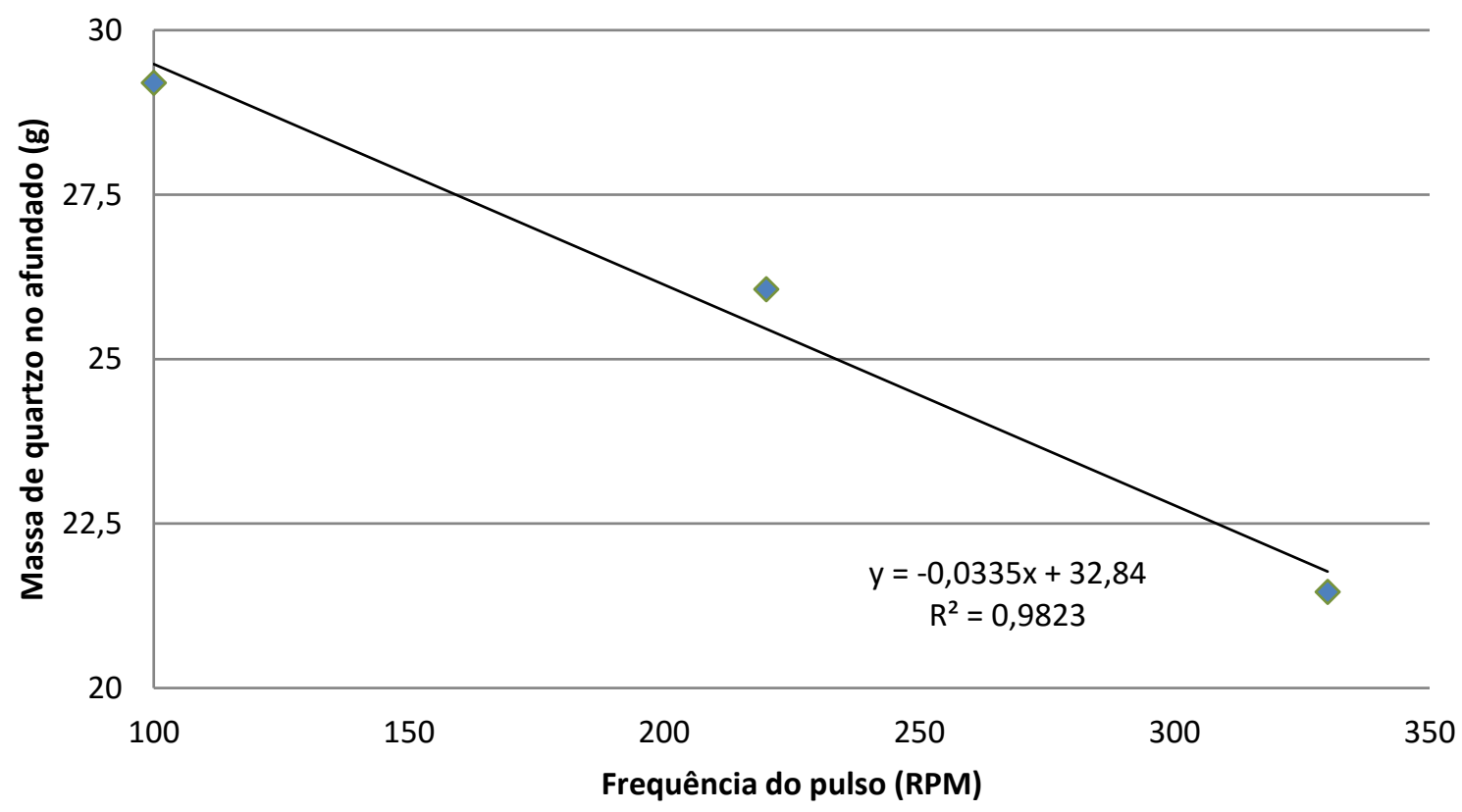

Figura 4: Massa de quartzo no material afundado em função da frequência dos pulsos do jigue para granulometria de $106 \mu \mathrm{m}$ (150\#).

Observa-se que o enriquecimento das amostras aumenta nas duas variáveis analisadas: 0 aumento da frequência de jigagem gera uma melhora na purificação do material; e também com a diminuição da granulometria há uma maior retirada do mineral leve (quartzo) das amostras testadas.

\section{CONCLUSÕES}

O estudo da jigagem para a retirada de materiais finos da amostra apresentou bons resultados no que se diz respeito de enriquecimento do material afundado. Mesmo com a granulometria mais grosseira e com frequência mais baixa, situação de pior cenário, houve um enriquecimento do material em quase $5 \%$. Nas condições mais favoráveis, que são com granulometria mais fina e frequência mais alta, vemos uma melhora no material de quase $20 \%$.

Estes resultados indicam que a jigagem é um processo válido na concentração de minerais quando se tem a necessidade de retirada de elementos mais leves dos materiais. Com isso podemos utilizar extrapolar os resultados e dizer que esse método possui parâmetros que indicam uma eficiência na separação da magnetita do minério de rocha fosfática proveniente das mineradoras da região de Catalão. Com a separação desse material vemos que é possível produzir concentrados de magnetita por jigagem, sendo possível a sua utilização na produção de ferrofluidos.

Como trabalhos futuros é necessário a realização de mais estudos com outras granulometrias e frequências, para melhor detalhamento destas variáveis, utilizando amostras diretas do rejeito das mineradoras no processo de jigagem, além de incluir outras variáveis no estudo como a vazão volumétrica de água e a espessura da camada de fundo. 


\section{AGRADECIMENTOS}

Os autores agradecem às agências de fomento CNPq, CAPES, FAPEG e FUNAPE pelo suporte financeiro, à Anglo American Fosfato do Brasil pela doação das amostras minerais que possibilitou a realização desta pesquisa e à Universidade Federal de Goiás.

\section{REFERÊNCIAS BIBLIOGRÁFICAS}

1. BRASIL. Departamento Nacional de Produção Mineral. Anuário Mineral Brasileiro. Brasília: DNPM, 2014.

2. BURT, R.O. Gravity Concentration Technology. Amsterdam: Elsevier,1984.

3. COSTA, T.C.C. Síntese de nanopartículas de magnetita via decomposição térmica em meio nãoaquoso. UFRN, 2013.

4. LINS, F.A.F. Concentração gravítica. In: DA LUZ, A.B; SAMPAIO, J.A; FRANÇA, S.C.A. Tratamento de Minérios. Rio de janeiro: CETEM/MCT, 2010. 5a Ed. Cap. 8. P. 329-366.

5. SAMPAIO, C.H; TAVARES, L.M.M. Beneficiamento Gravimétrico. Porto Alegre: Editora da UFRGS, 2005.

6. WILLS, B.; NAPPIER T. M. Mineral Processing Technology. 2006. 